\title{
Existence of Solutions for Sturm-Liouville Boundary Value Problems with Impulses
}

\author{
Qiaoluan Li, Shuang Zhang, Lina Zhou \\ College of Mathematics \& Information Science \\ Hebei Normal University, \\ Shijiazhuang, 050024, China
}

\author{
Weihua Jiang \\ (corresponding author) \\ School of Science, \\ Hebei University of Science and Technology, \\ Shijiazhuang, 050018,China
}

\begin{abstract}
In the paper, we investigate the Sturm-Liouville boundary value problem. By using fixed point methods, we establish sufficient conditions to guarantee the existence of solutions. At the end of the paper, two examples are given to illustrate our main results.
\end{abstract}

Keywords-Sturm-Liouville; Impulses; Existence

\section{INTRODUCTION}

Consider the impulsive differential equation with Sturm-Liouville boundary conditions

$$
\left\{\begin{array}{l}
\left(p(t) u^{\prime}(t)\right)^{\prime}+f(t, u, T u, S u)=0, t \in J, t \neq t_{j} \\
\Delta p\left(t_{j}\right) u\left(t_{j}\right)=I_{j}\left(u\left(t_{j}\right)\right), \\
-\Delta p\left(t_{j}\right) u^{\prime}\left(t_{j}\right)=J_{j}\left(u\left(t_{j}\right)\right), \quad j=1,2, \cdots n \\
\alpha u(0)-\beta p(0) u^{\prime}(0)=0, \\
\gamma u(1)+\delta p(1) u^{\prime}(1)=0 .
\end{array}\right.
$$

Where $p \in C[0,1], p(t)>0, J=[0,1]$,

$$
T u=\int_{0}^{t} k(t, s) u(s) d s, S u=\int_{0}^{1} k_{1}(t, s) u(s) d s, k \geq 0,
$$

$k_{1} \geq 0, \alpha, \beta, \gamma, \delta \geq 0, \Gamma=\beta \gamma+\alpha \delta+\alpha \gamma \int_{0}^{1} \frac{d \tau}{p(\tau)}>0$,

$$
f, I_{j}, J_{j} \text { are continuous, } \Delta z\left(t_{j}\right)=z\left(t_{j}^{+}\right)-z\left(t_{j}^{-}\right) \text {. }
$$

Recently, the research of impulsive initial and boundary value problems is extensive and there is increasing interest on the existence of impulsive differential equations. Numerous papers have been published on this class of equations and good results were obtained [1,3,6,7,10]. For instance, in 2008, Kaufmann [4] studied a second-order nonlinear differential equation subject to Sturm-Liouville type boundary conditions and impulsive conditions. The authors used Krasnoselskii's fixed point theorem to obtain the existence of solutions. In 2012, Wang [8] studied impulsive fractional differential equation. Some sufficient conditions for existence of the solutions are obtained by using fixed point methods.

This paper is organized as follows. In Section 2, we shall offer some basic definitions, preliminary results and Lemmas.

In section 3, we prove the main results. To illustrate our results, two examples are given in Section 4.

\section{PRELIMINARIES}

In order to prove our Theorems, we need the following definition and Lemmas.

Let $P C^{1}(J, R)=\left\{u: J \rightarrow R,\left.u\right|_{\left(t_{k}, t_{k+1}\right)},\left.u^{\prime}\right|_{\left(t_{k}, t_{k+1}\right)} \in C\left(t_{k}, t_{k+1}\right)\right.$, $\left.u\left(t_{k}^{-}\right)=u\left(t_{k}\right), \exists u^{\prime}\left(t_{k}^{-}\right), u\left(t_{k}^{+}\right), u^{\prime}\left(t_{k}^{+}\right)\right\} \quad$ with the norm $\|u\|=\max \left\{\sup _{t \in[0,1]}|u(t)|, \sup _{t \in[0,1]}\left|u^{\prime}(t)\right|\right\}$. A function $u$ is called a solution of Eq.(1.1) if $u \in P C^{1}(J, R)$ satisfies Eq.(1.1).

It is easy to know that $u$ is the solution of Eq.(1.1) if and only if $u$ satisfies the integral equation

$$
\begin{aligned}
u(t)=\int_{0}^{1} G(t, s) f(s, u, T u, S u) d s+\sum_{j=1}^{n} G\left(t, t_{j}\right) J_{j}\left(u\left(t_{j}\right)\right) \\
+\sum_{j=1}^{n} H\left(t, t_{j}\right) I_{j}\left(u\left(t_{j}\right)\right)
\end{aligned}
$$

Where

$$
G(t, s)=\frac{1}{\Gamma}\left\{\begin{array}{l}
\left(\delta+\gamma \int_{t}^{1} \frac{d \tau}{p(\tau)}\right)\left(\beta+\alpha \int_{0}^{s} \frac{d \tau}{p(\tau)}\right), 0 \leq s \leq t \leq 1, \\
\left(\beta+\alpha \int_{0}^{t} \frac{d \tau}{p(\tau)}\right)\left(\delta+\gamma \int_{s}^{1} \frac{d \tau}{p(\tau)}\right), 0 \leq t \leq s \leq 1,
\end{array}\right.
$$

and

$$
H(t, s)=\frac{1}{\Gamma}\left\{\begin{array}{l}
\frac{\alpha}{p(s)}\left(\delta+\gamma \int_{t}^{1} \frac{d \tau}{p(\tau)}\right), 0 \leq s<t \leq 1, \\
-\frac{\gamma}{p(s)}\left(\beta+\alpha \int_{0}^{t} \frac{d \tau}{p(\tau)}\right), 0 \leq t \leq s \leq 1 .
\end{array}\right.
$$

Further by [11] we know that

$$
\omega k_{2}(t) k_{2}(s) \leq G(t, s) \leq \frac{1}{\Gamma} k_{2}(s)\left(\text { or } k_{2}(t)\right)
$$

where $k_{2}(t)=\left(\beta+\alpha \int_{0}^{t} \frac{d \tau}{p(\tau)}\right)\left(\delta+\gamma \int_{t}^{1} \frac{d \tau}{p(\tau)}\right)$,

$$
\omega=\frac{\Gamma}{\left(\beta+\alpha \int_{0}^{1} \frac{d \tau}{p(\tau)}\right)\left(\delta+\gamma \int_{0}^{1} \frac{d \tau}{p(\tau)}\right)} .
$$

The following Lemmas are needed.

LEMMA 1.([2]) Let $X$ be a Banach space with $\Omega \subset X$ be closed and convex. Assume $U$ is a relatively open subset of 
$\Omega$ with $0 \in U$, and let $S: \bar{U} \rightarrow \Omega$ be a compact, continuous maps. Then either

(i) $S$ has a fixed point in $\bar{U}$, or

(ii) there exists $u \in \partial U$ and $v \in(0,1)$ with $u=v S u$.

LEMMA 2. ([5]) Let $M$ be a closed convex and nonempty subset of a Banach space $X$. Let $A, B$ be two operators such that

(i) $\quad A x+B y \in M$ whenever $x, y \in M$.

(ii) $A$ is a compact and continuous

(iii) $B$ is a contraction mapping.

Then there exits a $z \in M$ such that $z=A z+B z$.

LEMMA 3.([9]) Let $X$ be a Banach space and $W \subset P C(J, X)$. If the following conditions are satisfied:

(i) $W$ is uniformly bounded subset of $P C(J, X)$,

(ii) $W$ is equicontinuous in $\left(t_{k}, t_{k+1}\right), k=0,1,2 \cdots m$ where $t_{0}=0, t_{m+1}=1$,

(iii) $\mathrm{W}(\mathrm{t})=\left\{\mathrm{u}(\mathrm{t}): \quad u \in W, t \in J \backslash\left\{t_{1}, \ldots, t_{m}\right\}\right\}$, $W\left(t_{k}^{+}\right)=\left\{u\left(t_{k}^{+}\right): u \in W\right\}$ and $W\left(t_{k}^{-}\right)=\left\{u\left(t_{k}^{-}\right): u \in W\right\}$ are relatively compact set of $X$. Then $W$ is a relatively compact subset of $P C(J, X)$.

LEMMA 4.([8]) Let $X$ be a Banach spaces and $F: X \rightarrow X$ be a completely continuous operator. If the set

$E(F)=\{y \in X: y=\lambda F y$ for some $\lambda \in[0,1]\}$

is bounded, then $F$ has at least a fixed point.

\section{MAIN RESULTS}

We make the following assumptions:

(H1) There exists a positive function $g \in C[0,1]$ such that $f(t, u, T u, S u) \geq-g(t), t \in(0,1), u \in[0,+\infty)$.

(H2) $|f(t, u, v, w)-f(t, \bar{u}, \bar{v}, \bar{w})| \leq m(t)(|u-\bar{u}|+|v-\bar{v}|$ $+|w-\bar{w}|)$, where $m(t) \in C[0,1]$.

(H3) There exist $\mathrm{L}_{1}, \mathrm{~L}_{2}, \mathrm{M}_{1}, \mathrm{M}_{2}$ such that $\left\|I_{k}(u)-I_{k}(v)\right\| \leq L_{1}\|u-v\|, \quad\left\|I_{k}(u)\right\| \leq M_{1}$, $\left\|J_{k}(u)-J_{k}(v)\right\| \leq L_{2}\|u-v\|, \quad\left\|J_{k}(u)\right\| \leq M_{2}$.

(H4) There exists a constant $L_{3}$ such that $|f(t, u, T u, S u)| \leq L_{3}(1+|u|+|T u|+|S u|)$.

Assume $v(t)$ is the solution of the following equation

$$
\left\{\begin{array}{l}
\left(p(t) v^{\prime}(t)\right)^{\prime}+g(t)=0, t \in J, t \neq t_{j} \\
-\Delta p\left(t_{j}\right) v^{\prime}\left(t_{j}\right)=a_{j}, \quad j=1,2, \cdots n, \\
\alpha v(0)-\beta p(0) v^{\prime}(0)=0, \\
\gamma v(1)+\delta p(1) v^{\prime}(1)=0,
\end{array}\right.
$$

then $v$ satisfies

$$
v(t)=\int_{0}^{1} G(t, s) g(s) d s+\sum_{j=1}^{n} G\left(t, t_{j}\right) a_{j},
$$

where $\mathrm{a}_{j}$ will be defined in Theorem $1, \mathrm{G}(\mathrm{t}, \mathrm{s})$ is defined as (2.1).

Consider

$$
\left\{\begin{array}{l}
\left(p(t) u^{\prime}(t)\right)^{\prime}+f\left(t,(u-v)^{*}, T(u-v)^{*}, S(u-v)^{*}\right) \\
+g(t)=0, \quad t \in J, t \neq t_{j} \\
-\Delta p\left(t_{j}\right) u^{\prime}\left(t_{j}\right)=J_{j}\left(u\left(t_{j}\right)-v\left(t_{j}\right)\right)+a_{j}, \quad j=1,2, \cdots n \\
\alpha u(0)-\beta p(0) u^{\prime}(0)=0, \\
\gamma u(1)+\delta p(1) u^{\prime}(1)=0 .
\end{array}\right.
$$

where

$$
x^{*}(t)= \begin{cases}x(t), & x(t) \geq 0, \\ 0, & x(t)<0 .\end{cases}
$$

In the following Theorem 1, we shall prove (3.2) has a solution $u(t) \geq v(t)$ and $u(t)-v(t)$ is a nonnegative solution of Eq.(1.1).

THEOREM 1. Assume (H1) hold and $I_{j}=0$, $0 \leq J_{j}(x) \leq M_{2}$. There exist $R_{0}, a_{j}>0, j=1,2, \cdots, n$ such that $\int_{0}^{1} G(t, s) f(s, u, T u, S u) d s \geq 0$, for $t \in[0,1], 0 \leq u \leq R_{0}$, and

$$
\begin{aligned}
\Gamma R_{0}> & \int_{0}^{1} k_{2}(s) \max _{0 \leq s \leq 1,0 \leq z \leq R_{0}}(f(s, z, T z, S z)+g(s)) d s \\
& +M_{2} \sum_{j=1}^{n} k_{2}\left(t_{j}\right)+\sum_{j=1}^{n} k_{2}\left(t_{j}\right) a_{j} .
\end{aligned}
$$

Then Eq. (1.1) has at least one positive solution.

\section{PROOF. Let}

$$
\begin{aligned}
F u= & \int_{0}^{1} G(t, s)\left(f\left(s,(u-v)^{*}, T(u-v)^{*}, S(u-v)^{*}\right)+g(s)\right) d s \\
& +\sum_{j=1}^{n} G\left(t, t_{j}\right) J_{j}\left(u\left(t_{j}\right)-v\left(t_{j}\right)\right)+\sum_{j=1}^{n} G\left(t, t_{j}\right) a_{j} .
\end{aligned}
$$

We define $P=\left\{x \in P C^{1}(J, R): x(t) \geq \Gamma \omega k_{2}(t)\|x\|\right\}$.

It is easy to know $F u \geq 0$ for $u \in P$ and

$$
\begin{aligned}
F u \geq & \omega k_{2}(t)\left(\int _ { 0 } ^ { 1 } k _ { 2 } ( s ) \left(f\left(s,(u-v)^{*}, T(u-v)^{*}, S(u-v)^{*}\right)\right.\right. \\
& \left.+g(s)) d s+\sum_{j=1}^{n} k_{2}\left(t_{j}\right) J_{j}\left(u\left(t_{j}\right)-v\left(t_{j}\right)\right)+\sum_{j=1}^{n} k_{2}\left(t_{j}\right) a_{j}\right) .
\end{aligned}
$$

Furthermore

$$
\begin{aligned}
F u \leq & \frac{1}{\Gamma}\left(\int_{0}^{1} k_{2}(s)\left(f\left(s,(u-v)^{*}, T(u-v)^{*}, S(u-v)^{*}+g(s)\right) d s\right)\right. \\
& \left.+\sum_{j=1}^{n} k_{2}\left(t_{j}\right) J_{j}\left(u\left(t_{j}\right)-v\left(t_{j}\right)\right)+\sum_{j=1}^{n} k_{2}\left(t_{j}\right) a_{j}\right),
\end{aligned}
$$

then $F u \geq \omega k_{2}(t) \Gamma\|F u\|$. Hence $F(P) \subset P$.

Since $f$ and $J_{j}$ are continuous, we get $F$ is continuous. We will prove $F$ is uniformly bounded.

Let $D \subset P$ be bounded, i.e. there exists $L>0$ such that $\|u\| \leq L$ for $u \in D$.

$$
\text { Let } A=\max _{t \in[0,1], y \in[0, L]}|f(t, y, T y, S y)+g(t)| \text {, }
$$

$$
M=\max _{(t, s) \in[0,1] \times[0,1]} G(t, s),
$$

$|F u| \leq \frac{1}{\Gamma} \int_{0}^{1} k_{2}(s)\left(f\left(s,(u-v)^{*}, T(u-v)^{*}, S(u-v)^{*}\right)\right.$

$$
+g(s)) d s+M_{2} \sum_{j=1}^{n} G\left(t, t_{j}\right)+\sum_{j=1}^{n} G\left(t, t_{j}\right) a_{j}
$$




$$
\leq \frac{A}{\Gamma} \int_{0}^{1} k_{2}(s) d s+n M M_{2}+M \sum_{j=1}^{n} a_{j} .
$$

Therefore $F(D)$ is bounded.

It is easy to know $F: P \rightarrow P$ is equicontinuous. Hence $F$ is completely continuous.

Let $U=\left\{u \in P\right.$ and $\left.\|u\|<R_{0}\right\}$, notice that

$F: \bar{U} \rightarrow P C^{1}(J, R) \quad$ is continuous and completely continuous. Choose $u \in \bar{U}$ and $\lambda \in(0,1)$ such that $u=\lambda F u$. We claim that $\|u\| \neq R_{0}$. If not, then $\|u\|=R_{0}$.

$\|u\| \leq\|F U\|$

$$
\begin{aligned}
\leq & \frac{1}{\Gamma}\left(\int_{0}^{1} k_{2}(s) \max _{0 \leq s \leq 1,0 \leq z \leq R_{0}}\{f(s, z, T z, S z)+g(s)\} d s\right. \\
& \left.+M_{2} \sum_{j=1}^{n} k_{2}\left(t_{j}\right)+\sum_{j=1}^{n} k_{2}\left(t_{j}\right) a_{j}\right)
\end{aligned}
$$

which implies $\|u\| \neq R_{0}$. By the nonlinear alternative theorem of Leray-Schauder type, $\quad F$ has a fixed point $u \in \bar{U}$.

$$
\begin{aligned}
u(t) & =\int_{0}^{1} G(t, s) g(s) d s+\sum_{j=1}^{n} G\left(t, t_{j}\right) a_{j} \\
& +\sum_{j=1}^{n} G\left(t, t_{j}\right) J_{j}\left(u\left(t_{j}\right)-v\left(t_{j}\right)\right) \\
& +\int_{0}^{1} G(t, s) f\left(s,(u-v)^{*}, T(u-v)^{*}, S(u-v)^{*}\right) d s \\
& \geq v(t) .
\end{aligned}
$$

Let $x(t)=u(t)-v(t) \geq 0$. For $t \neq t_{j}$ we get

$$
\left(p(t) x^{\prime}(t)\right)^{\prime}=-f(t, x(t), T x(t), S x(t)),
$$$$
\text { and } \Delta p\left(t_{j}\right) x^{\prime}\left(t_{j}\right)=-J_{j}\left(x\left(t_{j}\right)\right) \text {. }
$$

Furthermore we obtain $\alpha x(0)-\beta p(0) x^{\prime}(0)=0$, and $\gamma x(1)+\delta p(1) x^{\prime}(1)=0$. So Eq.(1.1) has a positive solution. The proof is complete.

THEOREM 2. Assume that (H2) holds, $I_{j}=0$, $\left|J_{j}(u)-J_{j}(v)\right| \leq L_{4}|u-v|,\left\|J_{j}(u)\right\| \leq M_{2}, \quad \mathrm{M}<1$, where

$$
\begin{gathered}
M=\inf \left\{a>0: \int_{0}^{1} G(t, s) m(s)\left(k_{2}(s)+\int_{0}^{s} k(s, \tau) k_{2}(\tau) d \tau\right.\right. \\
\left.+\int_{0}^{1} k_{1}(s, \tau) k_{2}(\tau) d \tau\right) d s+L_{4} \sum_{j=1}^{n} G\left(t, t_{j}\right) k_{2}\left(t_{j}\right) \\
\left.\leq a k_{2}(t)\right\}
\end{gathered}
$$

then Eq.(1.1) has a unique continuous solution.

PROOF. Define $F: P C^{1}(J, R) \rightarrow P C^{1}(J, R)$ as follows:

$(F u)(t)=\int_{0}^{1} G(t, s) f(s, u, T u, S u) d s+\sum_{j=1}^{n} G\left(t, t_{j}\right) J_{j}\left(u\left(t_{j}\right)\right)$.

For $u \in P C^{1}(J, R)$, we have

$$
\begin{aligned}
& |(F u)(t)| \leq \int_{0}^{1} G(t, s)|f(s, u, T u, S u)-f(s, 0,0,0)| d s \\
& \quad+\int_{0}^{1} G(t, s)|f(s, 0,0,0)| d s+\sum_{j=1}^{n} G\left(t, t_{j}\right)\left|J_{j}\left(u\left(t_{j}\right)\right)\right| \\
& \leq \frac{k_{2}(t)}{\Gamma}\left\{\int_{0}^{1} m(s)(|u|+|T u|+|S u|) d s\right.
\end{aligned}
$$

$$
\left.+\int_{0}^{1}|f(s, 0,0,0)| d s+n M_{2}\right\} .
$$

So, $F$ maps $P C^{1}[0,1]$ into the following

$P C_{k_{2}}^{1}[0,1]=\left\{x \in P C^{1}[0,1]: \exists \alpha>0 \quad\right.$ such that $-\alpha k_{2}(t) \leq$

$$
\left.x(t) \leq \alpha k_{2}(t)\right\} \text {. }
$$

Define $\|x\|_{k_{2}}=\inf \left\{\alpha>0:-\alpha k_{2}(t) \leq x(t) \leq \alpha k_{2}(t)\right\}$.

We know $P C_{k_{2}}^{1}[0,1]$ is a subspace of $P C^{1}[0,1]$ and $P C_{k_{2}}^{1}[0,1]$ is an Banach space with the norm $\|\mathrm{x}\|_{k_{2}}$. Let $u, v \in P C_{k_{2}}^{1}[0,1]$,

$$
\begin{aligned}
& \quad|(F u)(t)-(F v)(t)| \\
& \leq\|u-v\|_{k_{2}}\left(\int _ { 0 } ^ { 1 } G ( t , s ) m ( s ) \left(k_{2}(s)+\int_{0}^{s} k(s, \tau) k_{2}(\tau) d \tau\right.\right. \\
& \left.\left.\quad+\int_{0}^{1} k_{1}(s, \tau) k_{2}(\tau) d \tau\right) d s+L_{4} \sum_{j=1}^{n} G\left(t, t_{j}\right) k_{2}\left(t_{j}\right)\right) \\
& \leq M k_{2}(t)\|u-v\|_{k_{2}} .
\end{aligned}
$$

So $\|F u-F v\|_{k_{2}} \leq M\|u-v\|_{k_{2}}$. From $M<1$, the operator $F$ is a contraction. By Banach's contraction principle, $F$ has a unique fixed point. The proof is complete.

$$
\begin{aligned}
& \text { THEOREM 3. Assume that (H2), } \\
& L:=\sup _{(t, s) \in[0,1] \times[0,1]}|H(t, s)|, \\
& \sup _{t \in[0,1]} \frac{1}{\Gamma} \int_{0}^{1} k_{2}(s) m(s)(1+T 1+S 1) d s=\eta_{1}<1, \\
& \frac{L_{2}}{\Gamma} \sum_{j=1}^{n} k_{2}\left(t_{j}\right)+n L L_{1}<1,
\end{aligned}
$$

then the Eq.(1.1) has at least one solution.

PROOF. Choose $B_{r}=\left\{u \in P C^{1}(J, R),\|u\| \leq r\right\}$, where

$r \geq \frac{\eta_{2}}{1-\eta_{1}}$

$\eta_{2}=\frac{1}{\Gamma} \int_{0}^{1} k_{2}(s)|f(s, 0,0,0)| d s+\frac{M_{2}}{\Gamma} \sum_{j=1}^{n} k_{2}\left(t_{j}\right)+n L M_{1}$,

and define on $B_{r}$ the operator $\Phi, \Psi$ by

$(\Phi u)(t)=\int_{0}^{1} G(t, s) f(s, u, T u, S u) d s$

and

$(\Psi u)(t)=\sum_{j=1}^{n} G\left(t, t_{j}\right) J_{j}\left(u\left(t_{j}\right)\right)+\sum_{j=1}^{n} H\left(t, t_{j}\right) I_{j}\left(u\left(t_{j}\right)\right)$.

Let us observe that if $u, v \in B_{r}$ then $\Phi u+\Psi v \in B_{r}$. Indeed

$|(\Phi u)+(\Psi v)|$

$\leq \int_{0}^{1} G(t, s)|f(s, u, T u, S u)-f(s, 0,0,0)| d s$

$+\int_{0}^{1} G(t, s)|f(s, 0,0,0)| d s+\sum_{j=1}^{n} G\left(t, t_{j}\right)\left|J_{j}\left(u\left(t_{j}\right)\right)\right|$

$+\sum_{j=1}^{n}\left|H\left(t, t_{j}\right) \| I_{j}\left(u\left(t_{j}\right)\right)\right|$

$\leq \frac{1}{\Gamma} \int_{0}^{1} k_{2}(s) m(s)(r+T r+S r) d s+\eta_{2}$

$\leq r \eta_{1}+\eta_{2} \leq r$.

It is easy to see that $\Psi$ is a contraction mapping. Since $f$ is continuous, we get $\Phi$ is continuous and $\|\Phi u\|<r$. 
It is easy to see that $\Phi$ is equicontinuous on interval $\left(t_{k}, t_{k+1}\right]$. So $\Phi$ is relatively compact on $B_{r}$. Hence by PCtype Arzela-Ascoli Theorem, $\Phi$ is compact on $B_{r}$. By Lemma 2, Eq.(1.1) has at least one solution on $J$. The proof is complete.

THEOREM 4. Assume (H3), (H4) hold and $B=\frac{L_{3}}{\Gamma} \int_{0}^{1} k_{2}(s)\left(1+\int_{0}^{s} k(s, \tau) d \tau+\int_{0}^{1} k_{1}(s, \tau) d \tau\right) d s<1$,

then the Eq.(1.1) has at least one solution.

PROOF. Define

$$
\begin{gathered}
F u(t)=\int_{0}^{1} G(t, s) f(s, u, T u, S u) d s+\sum_{j=1}^{n} G\left(t, t_{j}\right) J_{j}\left(u\left(t_{j}\right)\right) \\
+\sum_{j=1}^{n} H\left(t, t_{j}\right) I_{j}\left(u\left(t_{j}\right)\right)
\end{gathered}
$$

We first prove $F$ is continuous. Let $\left\{u_{m}\right\}$ be a sequence such that $u_{m} \rightarrow u$ in $P C^{1}(J, R)$. For $t \in J$,

$\left|\left(F u_{m}\right)(t)-(F u)(t)\right|$

$\leq \frac{1}{\Gamma} \int_{0}^{1} k_{2}(s) d s\left\|f\left(., u_{m}, T u_{m}, S u_{m}\right)-f(., u, T u, S u)\right\|$

$+\frac{1}{\Gamma} L_{2} \sum_{j=1}^{n} k_{2}\left(t_{j}\right)\left\|u_{m}-u\right\|+L L_{1} \sum_{j=1}^{n}\left\|u_{m}-u\right\|$,

where $L:=\sup _{(t, s) \in[0,1] \times[0,1]}|H(t, s)|$. By the continuous of $f$,

we get $F$ is continuous.

Second, we prove $F$ maps bounded sets into bounded set in $P C^{1}(J, R)$.

For any $\eta>0, B_{\eta}=\left\{u \in P C^{1}(J, R):\|u\| \leq \eta\right\}$,

$|(F u)(t)|$

$\leq \frac{L_{3}}{\Gamma} \int_{0}^{1} k_{2}(s)(1+|u|+|T u|+|S u|) d s$

$+\frac{M_{2}}{\Gamma} \sum_{j=1}^{n} k_{2}\left(t_{j}\right)+L n M_{1}$

$\leq \frac{L_{3}}{\Gamma} \int_{0}^{1}\left(1+\eta+\eta \int_{0}^{s} k(s, \tau) d \tau+\eta \int_{0}^{1} k_{1}(s, \tau) d \tau\right) k(s) d s$

$$
+\frac{M_{2}}{\Gamma} \sum_{j=1}^{n} k_{2}\left(t_{j}\right)+L n M_{1}:=l
$$

Which implies that $\|F u\| \leq l$.

It is easy to know that $F$ is equicontinuous on the interval $\left(t_{j}, t_{j+1}\right]$. By Lemma $3, F$ is continuous and complete continuous.

Let $E(F)=\left\{u \in P C^{1}(J, R), u=\lambda F(u), \lambda \in(0,1)\right\}$.

$|u(t)| \leq|F u(t)|$

$\leq \frac{L_{3}}{\Gamma} \int_{0}^{1} k_{2}(s) d s+\frac{L_{3}\|u\|}{\Gamma} \int_{0}^{1} k_{2}(s)\left(1+\int_{0}^{s} k(s, \tau) d \tau\right.$

$$
\left.+\int_{0}^{1} k_{1}(s, \tau) d \tau\right) d s+\frac{M_{2}}{\Gamma} \sum_{j=1}^{n} k_{2}\left(t_{j}\right)+n L M_{1}
$$

$:=A+\frac{L_{3}}{\Gamma} \int_{0}^{1} k_{2}(s)\left(1+\int_{0}^{s} k(s, \tau) d \tau+\int_{0}^{1} k_{1}(s, \tau) d \tau\right) d s\|u\|$, where $A=\frac{L_{3}}{\Gamma} \int_{0}^{1} k_{2}(s) d s+\frac{M_{2}}{\Gamma} \sum_{j=1}^{n} k_{2}\left(t_{j}\right)+n L M_{1}$. We obtain $\|u\| \leq \frac{A}{1-B}$. By Lemma 4, we deduce $F$ has a fixed point. The proof is complete.

\section{EXAMPLES}

In this section we give two examples to illustrate our main results.

EXAMPLE 1. Consider the following equation

$$
\left\{\begin{array}{l}
u^{\prime \prime}(t)+f(t, u, T u, S u)=0, t \in[0,1], t \neq \frac{1}{2} \\
-\Delta u^{\prime}\left(\frac{1}{2}\right)=\frac{1}{10}\left|\sin u\left(\frac{1}{2}\right)\right|, \\
u(0)-u^{\prime}(0)=0 \\
u(1)=0
\end{array}\right.
$$

where $f(t, u, T u, S u)=u^{2}(t)-2 u(t)+\frac{7}{8}+\int_{0}^{t} s u(s) d s$

$+\frac{1}{2}\left(\int_{0}^{1} s u(s) d s\right)^{2}$. Choose $g(t)=\frac{3}{16}$ for $u \geq 0$,

$$
f(t, u, T u, S u)+g(t) \geq(u-1)^{2}+\frac{1}{16}
$$

$$
+\int_{0}^{t} s u(s) d s+\frac{1}{2}\left(\int_{0}^{1} s u(s) d s\right)^{2}>0 .
$$

We choose $R_{0}=\frac{2}{3}, a_{1}=\frac{10}{27}$, it is easy to see that (3.3) holds.

By Theorem 1, Eq.(4.1) has at least one positive solution.

EXAMPLE 2. Consider the following equation

$$
\left\{\begin{array}{l}
u^{\prime \prime}(t)=-u-\int_{0}^{t} t \operatorname{su}(s) d s-\int_{0}^{1} \sin s u(s) d s, \quad t \in[0,1], t \neq \frac{1}{2} \\
\Delta u\left(\frac{1}{2}\right)=\frac{1}{2} \sin u\left(\frac{1}{2}\right) \\
-\Delta u^{\prime}\left(\frac{1}{2}\right)=\frac{1}{3} \cos u\left(\frac{1}{2}\right) \\
u(0)-u^{\prime}(0)=0 \\
u(1)=0
\end{array}\right.
$$

where $f(t, u, v, w)=u+v+w, T u=\int_{0}^{t} t s u(s) d s$,

$S u=\int_{0}^{1} \sin s u(s) d s$. It is easy to know that $(\mathrm{H} 2),(\mathrm{H} 3)$ hold, and $L=1, L_{1}=\frac{1}{2}, L_{2}=\frac{1}{3}$,

$\frac{1}{\Gamma} \int_{0}^{1} k_{2}(s) m(s)(1+T 1+S 1) d s \leq \frac{5-3 \cos 1}{6}<1$,

and (3.6) holds. By Theorem 3, the Eq. (4.2) has at least one solution. 


\section{REFERENCES}

[1] S. D. Akgol and A. Zefer, "Asymptotic integration of second-order impulsive differential equations”, Appl. Math. Lett.,Vol.76, pp. 1-7, 2018.

[2] C.Z. Bai, "Positive solutions for nonlinear fractional differential equations with coefficient that changes sign",Nonlinear Anal., Vol.64, pp. 677-685, 2006.

[3] D. Josef, "Positive solutions of nonlinear delayed differential equations with impulses", Appl.Math.Lett., Vol.72, pp. 16-22, 2017.

[4] E.R. Kaufmann, N. Kosmatov and Y.N. Raffoul, "A second-order boundary value problem with impulsive effects on an unbounded domain”, Nonlinear Anal.,Vol.69, pp. 2924-2929, 2008.

[5] M. A. Krasnoselskii, "Topological methods in the theory

[6] of nonlinear integral equations," New York: Pergamon Press, 1964.

[7] Q. Y. Li, Y. M. Zhou, F. Z. Cong and H. Liu, "Positive solutions to superlinear attractive singular impulsive differential equation," Appl. Math. Comput., Vol.338, pp. 822-827, 2018.

[8] Y. Tian, S. Shang and Q. Huo, "Antiperiodic solutions of fouthorder impulsive differential equations," Math. Methods Appl. Sci.,Vol.41, pp. 769-780, 2018.

[9] J. R. Wang, X. Z. Li and W. Wei, "On the natural solution of an impulsive fractional differential equation of order $q \in(1,2)$," Commun Nonlinear Sci Numer Simulat.,Vol.17, pp. 4384-4394, 2012.

[10] W. Wei, X. Xiang and Y. Peng, "Nonlinear impulsive integrodifferential equation of mixed type and optimal controls", Optimization., Vol.55, pp. 141-156, 2006.

[11] Z. L. Wei, "Periodic boundary value problems for second order impulsive integrodifferential equations of mixed type in Banach spaces,” J. Math. Anal. Appl.,Vol. 195, pp. 214-229, 1995.

[12] X. Zhang, L. Liu and H. Zou, "Eigenvalues of fourth-order singular Sturm-Liouville boundary value problems," Nonlinear Anal., Vol.68, pp. 384-392, 2008. 